\title{
MicroRNA-21 promotes neurite outgrowth by regulating PDCD4 in a rat model of spinal cord injury
}

\author{
YUQING JIANG ${ }^{*}$, SHUJIE ZHAO*, YIN DING, LUMING NONG, HAIBO LI, \\ GONGMING GAO, DONG ZHOU and NANWEI XU \\ Department of Orthopedics, Nanjing Medical University Affiliated Changzhou \\ No. 2 People's Hospital, Changzhou, Jiangsu 213000, P.R. China
}

Received May 19, 2016; Accepted January 3, 2017

DOI: $10.3892 / \mathrm{mmr} .2017 .6862$

\begin{abstract}
Altered expression levels of microRNA-21 (miRNA-21) have been observed in a series of pathological processes, including cancer and central nervous system injury; however, the involvement of miRNA-21 in the molecular pathophysiology of spinal cord injury (SCI) has not been well documented. The present study examined the expression levels of miRNA-21 and its predicted target genes, programmed cell death 4 (PDCD4) and phosphatase and tensin homolog (PTEN), in rats using quantitative polymerase chain reaction and western blotting to further understand the role of miRNA-21 and the mechanisms underlying repair following SCI. The present study demonstrated that compared with uninjured spinal cords, miRNA-21 expression levels were significantly downregulated in injured spinal cords 4 and $8 \mathrm{~h}$, and 1 day post-SCI, and were significantly upregulated after 3 and 7 days. Conversely, expression levels of PDCD4 and PTEN were significantly decreased at days 3 and 7 post-SCI compared with the control group. miRNA-21 overexpression in monolayer-cultured postnatal rat spinal cord neurons promoted neurite outgrowth and downregulated protein expression levels of PDCD4; however, PTEN protein expression levels were unaltered. To confirm that miRNA-21 directly targets PDCD4, a pRL-CMV luciferase reporter construct was used to detect miRNA-21 interactions with the PDCD4 3'-untranslated region. The results demonstrated that miRNA-21 decreased luciferase activity compared with a rat PDCD4 control reporter. The results of the present
\end{abstract}

Correspondence to: Dr Dong Zhou or Dr Nanwei Xu, Department of Orthopedics, Nanjing Medical University Affiliated Changzhou No. 2 People's Hospital, 29 Xing Long Road, Changzhou, Jiangsu 213000, P.R. China

E-mail: zhoudong1012@hotmail.com

E-mail: nanweixu1963@126.com

*Contributed equally

Key words: microRNA-21, neurite outgrowth, programmed cell death 4 , phosphatase and tensin homolog, spinal cord injury study suggested that increased miRNA-21 expression levels following SCI may promote the repair of injured spinal cords by inhibiting the expression of its target gene PDCD4.

\section{Introduction}

Micro (mi)RNAs are a class of single-stranded regulatory RNAs composed of 20-24 non-coding nucleotides that inhibit gene expression at the post-transcriptional level and regulate cell function $(1,2) ; 30 \%$ of mRNAs are regulated by miRNAs. Individual miRNAs are predicted to target dozens to hundreds of genes simultaneously $(3,4)$, and a single mRNA may contain multiple miRNA binding sites often targeted by separate miRNAs, which may increase regulation (5-7). miRNAs regulate gene expression by underlying mechanisms that are primarily dependent on the degree of miRNA sequence match to its cognate binding site within the target mRNA (8). miRNAs serve important roles in the regulation of numerous cellular and biological processes, including growth, development, differentiation, proliferation and apoptosis (9). Aberrant expression of miRNA-21 has been observed in a number of physiological and pathological processes; for example, downregulation of endogenous miRNA-21 in glioblastoma may initiate the caspase signaling pathway and increase apoptotic cell death (10), and upregulation of miRNA-21 in the cortex and hippocampus may promote repair following traumatic brain injury $(11,12)$. miRNA-21 contributes to pathophysiological processes directly and indirectly by regulating expression of target genes, including those involved in cell apoptosis, programmed cell death 4 (PDCD4), phosphatase and tensin homolog (PTEN), tissue inhibitor of metalloproteinase 3 and B-cell lymphoma 2 (13). A previous study demonstrated that miRNA-21 regulates cell apoptosis, proliferation and repair by regulating gene expression in cancer and following central nervous system (CNS) injury (14). Spinal cord injury (SCI) is a serious injury resulting in varying degrees of paraplegia. While numerous studies on SCI have centered on post-traumatic neuron cell repair and anti-apoptotic measures (15), the involvement of miRNA-21 in SCI pathophysiology remains to be investigated. The present study examined the expression levels of miRNA-21 and its predicted target genes PDCD4 and PTEN in rats following SCI. Additionally, the effect of miRNA-21 on neurite outgrowth and regulation of PDCD4 
expression levels was determined, and PDCD4 was demonstrated to be a target gene of miRNA-21. These results may further the understanding of the function of miRNA-21 in traumatic SCI.

\section{Materials and methods}

Spinal cord contusion injury. A total of 150 adult female Sprague-Dawley rats (weight, 200-250 g) were purchased from the Shanghai Animal Center, Chinese Academy of Sciences (Shanghai, China) and housed in a specific pathogen free facility with a constant humidity and temperature at $12 \mathrm{~h}: 12 \mathrm{~h}$ light:dark cycle with free access to food and water in the animal research facility in accordance with an approved protocol. The study protocol was approved by the Ethics committee at the Nanjing Medical University Affiliated Changzhou No. 2 People's Hospital (Changzhou, China). Rats in the injured group received SCI and were sacrificed (saline perfusion after $1 \%$ pentobarbital sodium anesthesia) at 4 or 8 h, or 1, 3 or 7 days following injury. Rats in the sham control group received laminectomy only. SCI was performed as previously reported (16). Briefly, rats were anesthetized with $1 \%$ pentobarbital sodium injected intraperitoneally $(50 \mathrm{mg} / \mathrm{kg}$; Sigma-Aldrich; Merck KGaA, Darmstadt, Germany) and kept warm on a heating pad. Following laminectomy at the T9 vertebra to expose the spinal cord (circular incision, $\sim 2.5-\mathrm{mm}$ in diameter), rats received moderate SCI at T9 using a New York University weight-drop device to drop a $10 \mathrm{~g}$ rod onto the dorsal surface of the cord from a height of $12.5 \mathrm{~mm}$. Rats received fluid infusion (crushed sterile food mixed with sterile distilled water) and antibiotics (gentamicin, $50 \mathrm{mg} / \mathrm{kg}$ ) for the first 3 days post-operation to minimize infection. Bladders were manually manipulated three times daily until normal voiding reflexes returned.

Protein extraction. The $10 \mathrm{~mm}$ long spinal cord segment containing the injury epicenter or a $10 \mathrm{~mm}$ long tissue at the same vertebral position from an uninjured spinal cord was dissected. Tissues were homogenized in $400 \mu \mathrm{l}$ radioimmunoprecipitation assay (RIPA) buffer (Invitrogen; Thermo Fisher Scientific, Inc., Waltham, MA, USA) to extract total protein. Ultrasonic cellular lysis was performed on ice for $\sim 5 \mathrm{~min}$ following the addition of RIPA buffer. The homogenate was incubated on ice for $30 \mathrm{~min}$ and subsequently centrifuged twice at $10,000 \mathrm{x} \mathrm{g}$ for $10 \mathrm{~min}$ at $4^{\circ} \mathrm{C}$. Protein concentrations were determined using a Bicinchoninic Acid Protein Quantification kit (Sangon Biotech Co., Ltd., Shanghai, China), and samples were stored at $-80^{\circ} \mathrm{C}$ at a concentration of $5 \mu \mathrm{g} / \mu 1$ until further use.

Western blotting. A total of $25 \mu \mathrm{g}$ extracted proteins per animal underwent $10 \%$ denaturing SDS-PAGE and were subsequently transferred onto polyvinylidene difluoride membranes $(0.08 \mathrm{~A}$, $3 \mathrm{~h})$. The membranes were blocked with $1 \%$ bovine serum albumin in TBS containing Tween-20 (TBST) for $30 \mathrm{~min}$ at $30^{\circ} \mathrm{C}$. Membranes were incubated with a 1:1,000 dilution of PDCD4 (9535), PTEN (5384) and $\beta$-actin (12620; all from Cell Signaling Technology, Inc., Danvers, MA, USA) rabbit monoclonal primary antibodies at $4^{\circ} \mathrm{C}$ overnight. Following three washes with TBST (10 min/wash), the membranes were incubated with a 1:5,000 dilution of a horseradish peroxidase-conjugated goat anti-rabbit IgG (GW0103S; Beijing ConWin Biotech, Co., Ltd., Beijing, China) for $1 \mathrm{~h}$ at $37^{\circ} \mathrm{C}$. Following a final wash, the membranes were developed using Western Lightning ${ }^{\circledR}$ Enhanced Chemiluminescence (34080; Invitrogen; Thermo Fisher Scientific, Waltham, MA, USA), and data were determined using Image Pro Plus software version 6.0 (Media Cybernetics, Inc., Rockville, MD, USA).

Total RNA extraction. Total RNA was extracted from spinal cord tissue using TRIzol ${ }^{\circledR}$ reagent (Sigma-Aldrich; Merck $\mathrm{KGaA}$ ) following the manufacturer's protocol. Briefly, $1 \mathrm{ml}$ TRIzol was used to homogenize the tissue in a $1.5 \mathrm{ml}$ RNase-free tube, followed by incubation at room temperature for 5-10 min. Chloroform (0.2 ml; Sigma-Aldrich; Merck $\mathrm{KGaA}$ ) was added and the tube was subsequently vortexed for $15 \mathrm{sec}$ and incubated at room temperature for 2-3 min. Following this, the tissue was centrifuged at $10,000 \times \mathrm{g}$ for $15 \mathrm{~min}$ at $4^{\circ} \mathrm{C}$. The aqueous layer was transferred to a new RNase-free tube and incubated with $0.5 \mathrm{ml}$ isopropyl alcohol at room temperature for $10 \mathrm{~min}$. The mixture was centrifuged at $10,000 \mathrm{x} \mathrm{g}$ for $10 \mathrm{~min}$ at $4^{\circ} \mathrm{C}$ to precipitate RNA, and $75 \%$ ethyl alcohol was used to wash the RNA pellet. Following this, the pellet was air-dried and resuspended in diethyl pyrocarbonate $\mathrm{H}_{2} \mathrm{O}$. RNA purity and concentration were determined spectrophotometrically at 230,260 and $280 \mathrm{~nm}$.

Quantitative polymerase chain reaction ( $q P C R$ ). The reverse transcription reaction was performed using the Prime-Script RT reagent kit (RR420A; Takara Biotechnology Co., Ltd., Dalian, China) and qPCR was performed using SYBR ${ }^{\circledR}$ Premix Ex Taq $^{\mathrm{TM}}$ kit (Tli RNaseH Plus; Takara Biotechnology Co., Ltd.) to detect expression levels of miRNA-21 at 4 or $8 \mathrm{~h}$, or 1,3 or 7 days post-SCI. Stage 1 was $95^{\circ} \mathrm{C}$ for $30 \mathrm{sec}(1 \mathrm{cycle})$, stage 2 was $95^{\circ} \mathrm{C}$ for $5 \mathrm{sec}$ and then 40 cycles at $60^{\circ} \mathrm{C}$ for $30 \mathrm{sec}$. The full-length sequence of miRNA-21 was 5'-TAGCTTATCAGACTGATGTTGA-3'. The sequence of qPCR primer was 5'-GAGGTATTCGCACTGGATACG-3' (Sangon Biotech Co., Ltd.). The U6 gene served as an endogenous control (forward, 5'-CTCGCTTCGGCAGCACA-3'; reverse, 5'-AACGCTTCA CGAATTTGCGT-3'). Quantitation threshold (Cq) values were determined using ABI Prism ${ }^{\circledR} 7900$ HT Fast Real-Time PCR system software version SDS2.5 (Thermo Fisher Scientific, Inc.). The relative quantity of miRNA-21 with respect to U6 was calculated using the $2^{-\Delta \Delta \mathrm{Cq}}$ method (17).

Primary spinal cord neuron culture. Spinal cord neurons of Sprague-Dawley rats at postnatal days 3-5 were purchased from the Shanghai Animal Center, Chinese Academy of Sciences (Shanghai, China) and were harvested and cultured in vitro using a modified method (18). Rats were anesthetized with diethyl ether (60297; Sinopharm Chemical Reagent Co., Ltd., Shanghai, China) and total spinal cord tissues were isolated. The complete spinal cord was placed in cold PBS $\left(-\mathrm{Ca}^{+2} / \mathrm{Mg}^{+2}\right)$; nerve roots and meninges were dissected using fine forceps, and the cord was sectioned into $0.5 \mathrm{~mm}$ pieces. The tissue was transferred to a $50 \mathrm{ml}$ tube and incubated in $0.1 \%$ trypsin-EDTA at $37^{\circ} \mathrm{C}$ for $30 \mathrm{~min}$ in a water bath, with agitation. The trypsin-EDTA was removed and replaced with $10 \mathrm{ml}$ Dulbecco's modified Eagle's medium/F-12 (DF12; 
11330-057), containing 10\% fetal bovine serum (10099-141), $1 \%$ N-2 supplement (17502-048), 2\% B27 (17504-044) and 1\% penicillin-streptomycin (15070-063) all from Thermo Fisher Scientific, Inc. Following this, the digested cells were gently and repeatedly pipetted using a $5 \mathrm{ml}$ pipette for $5 \mathrm{~min}$, filtered through a 100 mesh filter, and single cells in DF12 were plated onto coverslips coated with $200 \mu \mathrm{g} / \mathrm{ml}$ poly-L-lysine and $3 \mu \mathrm{g} / \mathrm{ml}$ laminin. The DF12 was replaced with Neurobasal medium (96\% Neurobasal-A; 10888-022; Thermo Fisher Scientific, Inc., 1\% N-2 supplement, 2\% B27 and 1\% penicillin-streptomycin) after $24 \mathrm{~h}$.

Recombinant adeno-associated virus (rAAV)-miRNA-21 transfection. To investigate the role of miRNA-21 on neurite outgrowth and its target genes, primary spinal cord neurons were transfected with an rAAV hU6-MCS-CMV-enhanced green fluorescent protein (EGFP; hU6) vector (Sangon Biotech Co., Ltd.) containing primary miRNA-21 (pri-miRNA-21), miRNA-21 inhibitor (in-miRNA-21) or miRNA negative control (nc-miRNA) sequences. rAAV plasmid production and purification were performed by GeneChem Co., Ltd (Shanghai, China). Following the manufacturer's protocol, $1.0 \times 10^{10}$ viral genomes of rAAV-pri-miRNA-21, rAAV-in-miRNA-21 or rAAV-nc-miRNA, or PBS as a control, were added to the cultured neurons 1 day post-plating.

Neurite outgrowth assay. Spinal cord neurons were transfected with rAAV-pri-miRNA-21, rAAV-in-miRNA-21 or rAAV-nc-miRNA at day 1 . At day 5, neurons were fixed in $4 \%$ paraformaldehyde and stained with a rabbit anti- $\beta$-III-tubulin antibody (ab11270; 1:1,000; Abcam, Cambridge, UK). Neurites co-stained with goat anti-rabbit rhodamine (R415; 1:100; Thermo Fisher Scientific, Inc.) and EGFP (PA1-18401; Thermo Fisher Scientific, Inc.) were observed under a fluorescent microscope (Leica Microsystems GmbH, Wetzlar, Germany) with representative photomicrographs being captured throughout. The longest neurite length for each of the first 100 neurons encountered during scanning (regardless of size and number of neurites) was measured using the Leica QW in software version 3 image analysis program (Leica Microsystems $\mathrm{GmbH}$ ). Neurite outgrowth analyses were performed in quadruplicate.

Luciferase reporter assay. Wild-type (wt; 5'-GAGAUGGAA UUUUAUGUAATT-3') and mutant (mt; 5'-UUACAUAAA AUUCCAUCUCCA-3') PDCD4 3'-untranslated regions (UTRs) were subcloned into the pRL-CMV miRNA expression vector containing Renilla luciferase (Promega Corporation, Madison, WI, USA) to generate wt-PDCD4 and mt-PDCD4. HEK293 human embryonic kidney cells (Cell bank of Chinese Academy of Sciences, Shanghai, China) were cultured in 24-well plates with Dulbecco's modified Eagle's medium (11995065; Thermo Fisher Scientific, Inc.) with 10\% FBS for $24 \mathrm{~h}$ and subsequently transfected with $200 \mathrm{ng}$ wt-PDCD4, mt-PDCD4 or $100 \mathrm{ng}$ empty pRL-CMV vector, and $10 \mu \mathrm{M}$ synthetic miRNA-21 mimic or miRNA NC (Sangon Biotech Co., Ltd.) using Lipofectamine 2000 ${ }^{\mathrm{TM}}$ (Thermo Fisher Scientific, Inc.) according to the manufacturer's protocol. Firefly and Renilla luciferase activities were measured $48 \mathrm{~h}$ post-transfection using the dual-luciferase reporter assay system. The
Renilla luciferase values were normalized to firefly luciferase values.

Statistical analysis. Statistical analysis of the experimental data was conducted using SPSS software version 17.0 (SPSS, Inc., Chicago, IL, USA). All data are presented as the mean \pm standard deviation. One-way analysis of variance followed by Tukey's post hoc test was used to analyze the differences in gene expression between groups. $\mathrm{P}<0.05$ was considered to indicate a statistically significant difference.

\section{Results}

miRNA-21, PDCD4 and PTEN expression profiles following $S C I$. The present study investigated the expression levels of miRNA-21 for 1 week following SCI by qPCR. miRNA-21 expression levels reduced 1 day following injury, and increased at days 3 and 7. As presented in Fig. 1A, miRNA-21 expression levels at 4 and $8 \mathrm{~h}$, and 1 day post-SCI were significantly reduced compared with the control group $(\mathrm{P}<0.05)$. miRNA-21 expression levels gradually increased at days 3 and 7 post-SCI, and were significantly increased compared with uninjured spinal cords at the 2 time points $(\mathrm{P}<0.05)$. To elucidate the role of miRNA-21 in SCI, the present study further analyzed the expression levels of PDCD4 (the predicted target gene of miRNA-21) at various time points following SCI to determine if there was a correlation with altered miRNA-21 expression. Western blot analysis (Fig. 1B) revealed that the expression levels of PDCD4 significantly decreased 3 and 7 days following injury compared with the uninjured group ( $\mathrm{P}<0.05$; Fig. $1 \mathrm{C})$. Additionally, PTEN expression levels at days 3 and 7 days following injury were reduced compared with control spinal cords $(\mathrm{P}<0.05$; Fig. 1D). The western blot analysis results suggested that miRNA-21-regulated PTEN activity may have an impact on cell survival and apoptosis.

miRNA-21 promotes neurite outgrowth and regulates PDCD4 expression levels. To examine the effect of miRNA-21 on neurite outgrowth, the present study overexpressed miRNA-21 in monolayer cultures of postnatal rat spinal cord neurons. Transfection efficiency of miRNA-21 in dissected neurons was determined by EGFP expression from the hU6-MCS-CMV-EGFP rAAV vector. In neuron cultures transfected with in-miRNA-21, neurons produced short or no neurites. By contrast, miRNA-21 overexpressing neurons demonstrated a significant increase in neurite outgrowth compared with control or nc-miRNA groups (Fig. 2A). Quantification of the longest fiber outgrowth from transfected neurons indicated that miRNA-21 significantly increased the mean length of the longest neurite compared with the control and nc-miRNA groups $(234 \pm 7.1,121 \pm 3.9$ and $109 \pm 4.7 \mathrm{~mm}$, respectively; $\mathrm{P}<0.05$; Fig. $2 \mathrm{~B}$ ). These data demonstrated that overexpression of miRNA-21 promotes neurite outgrowth in postnatal rat spinal cord neurons by increasing neurite extension.

It was subsequently investigated whether miRNA-21 reduced PDCD4 and PTEN protein expression levels in postnatal spinal cord neurons. As revealed by western blot analysis (Fig. 2C), compared with the control group, no significant differences were observed in PTEN protein expression levels, 


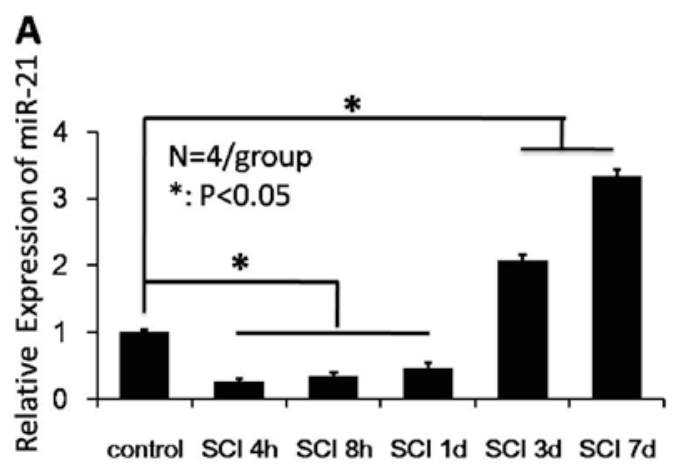

\section{B}
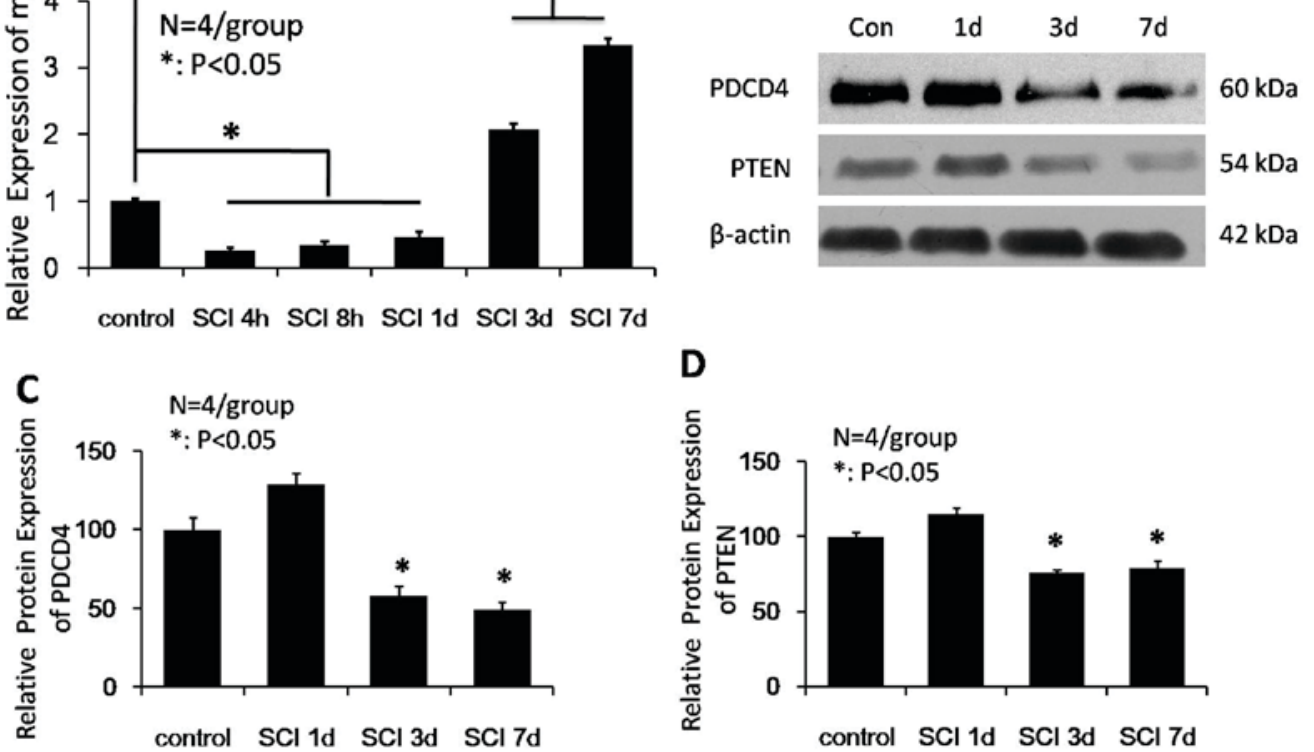

Figure 1. Altered expression of miR-21, PDCD4 and PTEN following SCI. (A) miR-21 expression levels were significantly reduced 4 and 8 h, and 1 day post-SCI compared with the control group, whereas miR-21 expression levels were significantly increased at days 3 and 7 post-SCI compared with the control group. (B) Representative western blot images and quantification of (C) PDCD4 and (D) PTEN protein expression levels. The expression levels of PDCD4 and PTEN were decreased significantly at days 3 and 7 post-SCI compared with the control group. Data are expressed as the mean \pm standard deviation ( $\mathrm{n}=4$ /group). "P<0.05 vs. control group. miR-21, microRNA-21; SCI, spinal cord injury; PDCD4, programmed cell death 4; PTEN, phosphatase and tensin homolog; con, control.
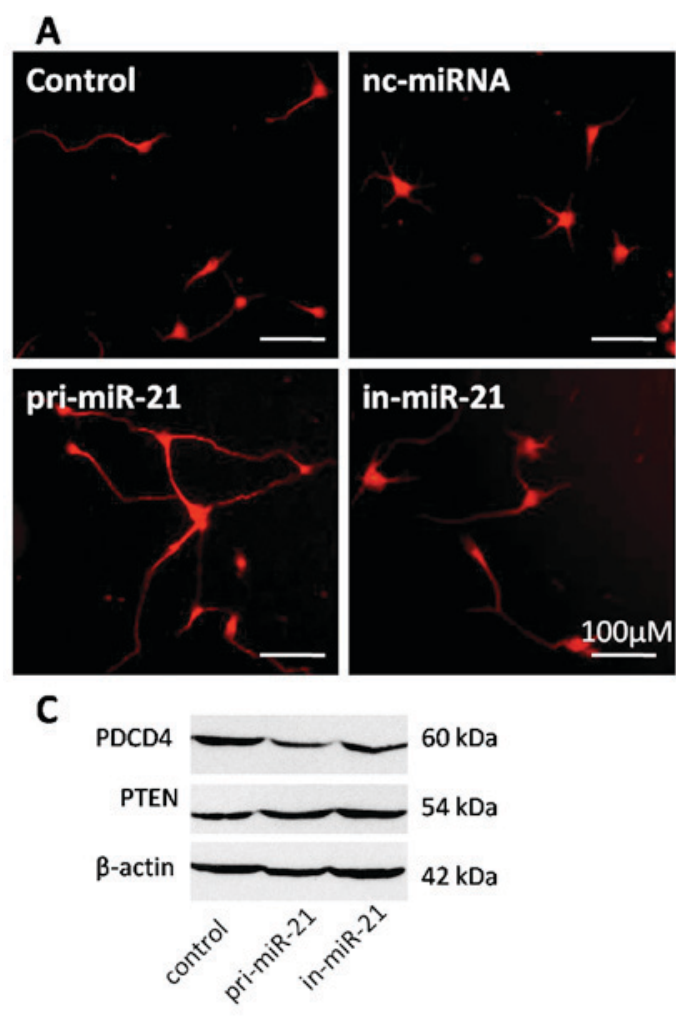

B

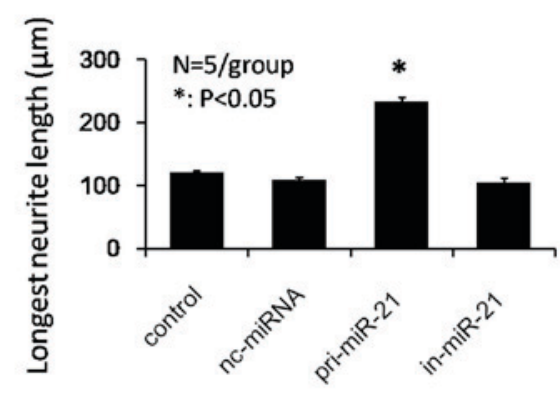

D

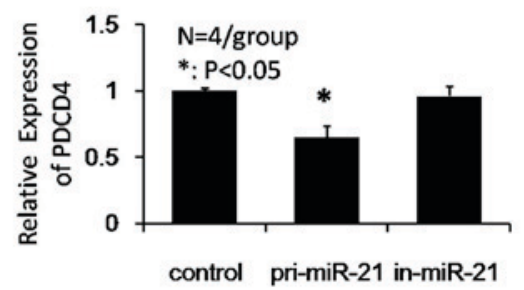

Figure 2. miR-21 promotes neurite outgrowth in rat spinal cord neurons. (A) Neurite outgrowth (detected by $\beta$-III tubulin in red) observed in control or miR-21-transfected spinal cord neurons. Scale bar, $100 \mu \mathrm{m}$. (B) miR-21 increased the mean length of the longest neurite (per neuron) compared with the control group ( $\mathrm{n}=5 /$ group). (C) Representative western blot images and (D) quantification of PDCD4 protein expression levels following transfection with pri-miR-21 or in-miR-21. $\beta$-actin served as an internal control. miR-21 significantly downregulated the expression levels of PDCD4. Data are expressed as the mean \pm standard deviation ( $\mathrm{n}=4$ /group). ${ }^{\mathrm{P}}<0.05$ vs. control group. miR-21, microRNA-21; PDCD4, programmed cell death 4; PTEN, phosphatase and tensin homolog; pri, primary; in, inhibitor; nc, negative control. 
A

\begin{tabular}{|c|c|}
\hline PDCD4 & \\
\hline Hsa & 5' - - GAAUAUUCUAAUAAGCUACC 3' \\
\hline Mmu & 5' U -GGGUGUUCUGAUAAGCUA - - 3' \\
\hline Rno & 5' UGGGGUGUUCUGAUAAGCUA - - 3' \\
\hline
\end{tabular}

B

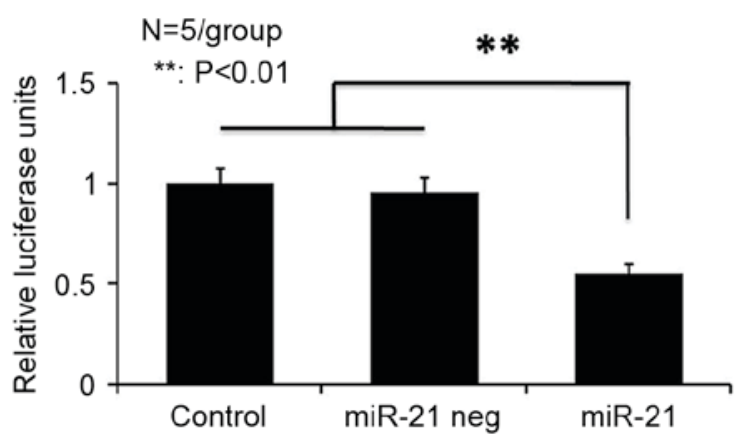

Figure 3. miR-21 targets the PDCD4 gene. (A) Alignment of the 3'-UTR sequence of PDCD4 in three species with miR-21, highlighting the fully conserved sequence in bold. (B) Relative luciferase expression in rat PDCD4 luciferase reporters co-transfected with miR-21-expressing constructs in HEK293 cells. miR-21 decreased luciferase activity in the miR-21-transfected group by $45 \pm 5.1 \%$ compared with the control and negative control groups. Data are expressed as the mean \pm standard deviation ( $\mathrm{n}=5 /$ group). ${ }^{* *} \mathrm{P}<0.01$. miR-21, microRNA-21; PDCD4, programmed cell death 4; neg, negative control.

whereas there was a 35\% decrease in PDCD4 expression levels ( $\mathrm{P}<0.05$; Fig. 2D).

PDCD4 is the target gene of miRNA-21. miRbase (www .mirbase.org) indicates that miRNA-21 has a high-energy binding site at 281-288 bp in human, mouse and rat PDCD4 3'-UTRs (Fig. 3A). To determine whether miRNA-21 directly targeted PDCD4, the present study cloned the 3'-UTR of rat PDCD4 downstream from the pRL-CMV luciferase reporter. Cotransfection of wt-PDCD4, mt-PDCD4 or empty pRL-CMV vectors along with an miRNA-21 mimic revealed that miRNA-21 decreased luciferase activity in wt-PDCD4-transfected cells by $45 \pm 5.1 \%$, compared with mt-PDCD 4 and negative control (miRNA-21 neg) neurons (Fig. 3B). Taken together, the western blot analysis and luciferase reporter assay results indicated that miRNA-21 binds directly to the 3'-UTR of rat PDCD4 mRNA and downregulates PDCD4 protein expression levels in postnatal spinal cord neurons.

\section{Discussion}

miRNAs are a class of endogenous noncoding small RNAs that negatively regulate gene expression at the post-transcriptional level by binding to the 3'-UTR of target mRNAs, leading to their translational inhibition or degradation. In humans, $20-30 \%$ of protein-coding genes are regulated by miRNAs $(5,19,20)$. Following SCI, a primary mechanical injury causes considerable alterations in gene expression, which leads to more serious secondary damage, mediated by multiple injury processes $(21,22)$. Previous studies have suggested that miRNAs are expressed specifically in the CNS and may regulate downstream protein expression levels by redistributing and altering expression levels in response to CNS injury, which may eventually affect repair and regeneration in the CNS, and the pathogenesis of secondary injury following SCI $(23,24)$. Recent investigations have focused on the role of miRNAs in SCI.

miRNA-21 has previously been identified in the spinal cord (25-27). A previous study demonstrated that overexpression of miRNA-21 in astrocytes caused a decrease in cell size and glial fibrillary acidic protein expression levels, suggesting a potential role for miRNA-21 in regulating astrogliosis following SCI (28). Furthermore, miRNA-21 has been suggested to be a potential therapeutic target for manipulating gliosis and enhancing functional outcome by regulating astrocytic hypertrophy and glial scar progression following SCI (25). One study reported that in a rat SCI model, exercise elevated expression levels of miRNA-21 and decreased expression levels of miRNA-199a-3p, which correlated with significant alterations in mRNA expression levels of their target genes PTEN and mammalian target of rapamycin, respectively (29). Given the potential role for miRNA-21 in SCI, the present study investigated miRNA-21 expression levels at 4 and $8 \mathrm{~h}$, and 1, 3 and 7 days following injury, and identified that its expression levels were significantly decreased during the early phase of injury: 4 and $8 \mathrm{~h}$ and 1 day post-SCI. It has previously been reported that acute SCI may result in extensive hemorrhage around the central canal and regional gray matter, with progressive neural degeneration and necrosis during the first $8 \mathrm{~h}$ post-injury, which triggers apoptotic cell death to limit the release of protease and toxic molecules (30). Secondary damage caused by tissue edema, hemorrhage, microcirculation disturbance and release of oxygen free radicals following acute neuron necrosis resulted in the migration of hypertrophic reactive astrocytes and microglial cells adjacent to the substantia nigra into the lesion sites, where they served important roles in the reparative process. A previous study indicated that overexpression of miRNA-21 may protect against ischemic neuronal death; therefore, miRNA-21 may be a potential therapeutic molecule for the treatment of stroke (31). Axotomy-induced miRNA-21 has previously been reported to promote axon growth in adult dorsal root ganglion neurons (32). The results of the present study suggested that downregulation of miRNA- 21 during the early phase of injury may contribute to the clearance of damaged debris from the lesion and facilitate the repair and regeneration of neurons and neural precursor cells bordering the lesion. The present study additionally demonstrated that miRNA-21 expression levels were significantly upregulated 3 days post-SCI, with peak expression levels at day 7. As astrocytic hypertrophy in the glial injury response may impede communication between proximal and distal nerve fibers in the lesion (33), these observations suggested that upregulation of miRNA-21 at a later phase of injury may contribute to the reparative process by inhibiting the migration of hypertrophic astrocytes and apoptosis of neural cells.

To date, the genes targeted by miRNA- 21 that contribute to cell survival and apoptosis remain elusive. A previous study demonstrated that miRNA-21 expression levels were 
significantly upregulated in the spinal cord following passive exercise in rats (34), indicating that miRNA-21 may function as an anti-apoptotic factor by inhibiting the expression levels of the pro-apoptotic factors PDCD4 and PTEN. In breast cancer cells, PDCD4 was revealed to be regulated and targeted by miRNA-21 (35). To further examine the role of endogenous miRNA-21 in SCI, the present study investigated the expression levels of the miRNA-21 targets PDCD4 and PTEN following injury. miRNA-21 has previously been demonstrated to serve a protective role in limiting secondary cell death following SCI, which may have been the result of its regulation of pro-apoptotic genes (26); this was consistent with the results of the present study. A negative correlation would indicate that miRNA-21 may block glial scar progression by regulating apoptotic factors and thus improve post-injury repair. The present study demonstrated that PDCD4 expression levels decreased 3 and 7 days post-injury. Similarly, PTEN expression levels were markedly downregulated at days 3 and 7 post-SCI. Taken together, these results suggested that the increased expression levels of miRNA-21 resulted in reduced expression levels of the target genes. Additionally, the present study demonstrated that miRNA-21 promoted spinal cord neurite outgrowth at postnatal days 3-5 in rats and inhibited protein expression of PDCD4 in cultured neurons in vitro. Luciferase reporter assays confirmed that PDCD4 is a target gene of mi-R21. These results thus suggested that miRNA-21 may repress glial scar progression and improve spinal cord repair by inhibiting the expression of the pro-apoptotic PDCD4 gene in the secondary phase of SCI.

Following SCI, a diverse set of cytokines is involved in the complex processes of pathogenesis and repair. Alterations in miRNAs following SCI may influence the biological repair process, including cell apoptosis, inflammation, glial scar formation and nerve fiber regeneration. The present study observed that increased miRNA-21 expression levels negatively regulate PDCD4 gene expression, thus inhibiting factors detrimental to nerve regeneration and repair and creating a more conducive environment for repair around the lesion sites. SCI commonly occurs among children and young adults and has a high rate of morbidity and mortality (36). Studies investigating the role of miRNA-21 on pathophysiological processes following SCI remain limited. The results of the present study facilitate a greater understanding of the role of miRNA-21 and the mechanisms underlying regeneration following SCI, which may be of significant value for the clinical treatment of nervous system injury.

\section{Acknowledgements}

The authors would like to thank Dr Hai-bin Xia (Institute of Biological Science, Shaanxi Normal University, Xi'an, China) for his individual support and help with this study. The present study was supported by the H-level Medical Talents Training Project (grant no. 2016CZBJ033).

\section{References}

1. Fire A, Xu S, Montgomery MK, Kostas SA, Driver SE and Mello CC: Potent and specific genetic interference by double-stranded RNA in Caenorhabditis elegans. Nature 391: 806-811, 1998
2. Griffiths-Jones S, Saini HK, van Dongen S and Enright AJ: miRBase: Tools for microRNA genomics. Nucleic Acids Res 36 (Database issue): D154-D158, 2008

3. Baek D, Villén J, Shin C, Camargo FD, Gygi SP and Bartel DP: The impact of microRNAs on protein output. Nature 455: 64-71, 2008.

4. Liu Q, Fu H, Sun F, Zhang H, Tie Y, Zhu J, Xing R, Sun Z and Zheng X: miR-16 family induces cell cycle arrest by regulating multiple cell cycle genes. Nucleic Acids Res 36: 5391-5404, 2008.

5. Fazekas D, Koltai M, Türei D, Módos D, Pálfy M, Dúl Z, Zsákai L, Szalay-Bekő M, Lenti K, Farkas IJ, et al: SignaLink 2-a signaling pathway resource with multi-layered regulatory networks. BMC Syst Biol 7: 7, 2013.

6. Kuzin A, Kundu M, Brody T and Odenwald WF: The Drosophila nerfin-1 mRNA requires multiple microRNAs to regulate its spatial and temporal translation dynamics in the developing nervous system. Dev Biol 310: 35-43, 2007.

7. Stark A, Brennecke J, Bushati N, Russell RB and Cohen SM: Animal MicroRNAs confer robustness to gene expression and have a significant impact on 3'UTR evolution. Cell 123: 1133-1146, 2005.

8. Bartel DP and Chen CZ: Micromanagers of gene expression: The potentially widespread influence of metazoan microRNAs. Nat Rev Genet 5: 396-400, 2004.

9. Kloosterman WP and Plasterk RH: The diverse functions of microRNAs in animal development and disease. Dev Cell 11: 441-450, 2006.

10. Chan JA, Krichevsky AM and Kosik KS: MicroRNA-21 is an antiapoptotic factor in human glioblastoma cells. Cancer Res 65: 6029-6033, 2005.

11. Lei P, Li Y, Chen X, Yang S and Zhang J: Microarray based analysis of microRNA expression in rat cerebral cortex after traumatic brain injury. Brain Res 1284: 191-201, 2009.

12. Redell JB,Zhao J and Dash PK: Altered expression of miRNA-21 and its targets in the hippocampus after traumatic brain injury. J Neurosci Res 89: 212-221, 2011.

13. Talotta F, Cimmino A, Matarazzo MR, Casalino L, De Vita G, D'Esposito M, Di Lauro R and Verde P: An autoregulatory loop mediated by miR-21 and PDCD4 controls the AP-1 activity in RAS transformation. Oncogene 28: 73-84, 2009.

14. Põlajeva J, Swartling FJ, Jiang Y, Singh U, Pietras K, Uhrbom L, Westermark B and Roswall P: miRNA-21 is developmentally regulated in mouse brain and is co-expressed with SOX2 in glioma. BMC Cancer 12: 378, 2012.

15. Donnelly DJ and Popovich PG: Inflammation and its role in neuroprotection, axonal regeneration and functional recovery after spinal cord injury. Exp Neurol 209: 378-388, 2008.

16. Dou F, Huang L, Yu P, Zhu H, Wang X, Zou J, Lu P and Xu XM: Temporospatial expression and cellular localization of oligodendrocyte myelin glycoprotein (OMgp) after traumatic spinal cord injury in adult rats. J Neurotrauma 26: 2299-2311, 2009.

17. Livak KJ and Schmittgen TD: Analysis of relative gene expression data using real-time quantitative PCR and the 2(-Delta Delta C(T)) Method. Method 25: 402-408, 2001.

18. Montoya-Gacharna JV, Sutachan JJ, Chan WS, Sideris A Blanck TJ and Recio-Pinto E: Preparation of adult spinal cord motor neuron cultures under serum-free conditions. Methods Mol Biol 846: 103-116, 2012.

19. Abe M and Bonini NM: MicroRNAs and neurodegeneration: Role and impact. Trends Cell Biol 23: 30-36, 2013.

20. Krichevsky AM: MicroRNA profiling: From dark matter to white matter, or identifying new players in neurobiology. ScientificWorldJournal 7: 155-166, 2007.

21. Baptiste DC and Fehlings MG: Update on the treatment of spinal cord injury. Prog Brain Res 161: 217-233, 2007.

22. Zai LJ, Yoo S and Wrathall JR: Increased growth factor expression and cell proliferation after contusive spinal cord injury. Brain Res 1052: 147-155, 2005

23. Bak M, Silahtaroglu A, Møller M, Christensen M, Rath MF, Skryabin B, Tommerup N and Kauppinen S: MicroRNA expression in the adult mouse central nervous system. RNA 14: 432-444, 2008.

24. Medina PP and Slack FJ: Inhibiting microRNA function in vivo. Nat Methods 6: 37-38, 2009.

25. Bhalala OG, Pan L, Sahni V, McGuire TL, Gruner K, Tourtellotte WG and Kessler JA: microRNA-21 regulates astrocytic response following spinal cord injury. J Neurosci 32: 17935-17947, 2012.

26. Hu JZ, Huang JH, Zeng L, Wang G, Cao M and Lu HB: Anti-apoptotic effect of microRNA-21 after contusion spinal cord injury in rats. J Neurotrauma 30: 1349-1360, 2013. 
27. Liu NK, Wang XF, Lu QB and Xu XM: Altered microRNA expression following traumatic spinal cord injury. Exp Neurol 219: 424-429, 2009.

28. Sahni V, Mukhopadhyay A, Tysseling V, Hebert A, Birch D Mcguire TL, Stupp SI and Kessler JA: BMPR1a and BMPR1b signaling exert opposing effects on gliosis after spinal cord injury. J Neurosci 30: 1839-1855, 2010.

29. Liu G, Detloff MR, Miller KN, Santi L and Houlé JD: Exercise modulates microRNAs that affect the PTEN/mTOR pathway in rats after spinal cord injury. Exp Neurol 233: 447-456, 2012.

30. Liu XZ, Xu XM, Hu R, Du C, Zhang SX, McDonald JW, Dong HX, Wu YJ, Fan GS, Jacquin MF, et al: Neuronal and glial apoptosis after traumatic spinal cord injury. J Neurosci 17: 5395-5406, 1997.

31. Buller B, Liu X, Wang X, Zhang RL, Zhang L, Hozeska-Solgot A, Chopp $\mathrm{M}$ and Zhang ZG: MicroRNA-21 protects neurons from ischemic death. FEBS J 277: 4299-4307, 2010.
32. Strickland IT, Richards L, Holmes FE, Wynick D, Uney JB and Wong LF: Axotomy-induced miR-21 promotes axon growth in adult dorsal root ganglion neurons. PLoS One 6: e23423, 2011.

33. Liu NK, Zhang YP, Titsworth WL, Jiang X, Han S, Lu PH, Shields CB and Xu XM: A novel role of phospholipase A2 in mediating spinal cord secondary injury. Ann Neurol 59: 606-619, 2006.

34. Liu G, Keeler BE, Zhukareva V and Houlé JD: Cycling exercise affects the expression of apoptosis-associated microRNAs after spinal cord injury in rats. Exp Neurol 226: 200-206, 2010.

35. Frankel LB, Christoffersen NR, Jacobsen A, Lindow M, Krogh A and Lund AH: Programmed cell death 4 (PDCD4) is an important functional target of the microRNA miR-21 in breast cancer cells. J Biol Chem 283: 1026-1033, 2008.

36. Boltshauser E: Spnal cord injury in the child and young adult. Neuropediatrics 2015 (Epub ahead of print). 